\title{
Pion beta decay and Cabibbo-Kobayashi-Maskawa unitarity
}

\author{
Andrzej Czarneckiఠ \\ Department of Physics, University of Alberta, Edmonton, Alberta T6G 2E1, Canada \\ William J. Marciano \\ Department of Physics, Brookhaven National Laboratory, Upton, New York 11973, USA
}

Alberto Sirlin $\odot$

Department of Physics, New York University, 726 Broadway, New York, New York 10003, USA

(Received 22 November 2019; revised manuscript received 2 February 2020; accepted 30 April 2020; published 27 May 2020)

Pion beta decay, $\pi^{+} \rightarrow \pi^{0} e^{+} \nu(\gamma)$, provides a theoretically clean $\pm 0.3 \%$ determination of the CabibboKobayashi-Maskawa matrix element $V_{\text {ud }}$. Although impressive, that result falls short of super-allowed nuclear beta decays where an order of magnitude better precision already exists. Here, we advocate a new strategy for utilizing pion beta decay, based on its utility in determining $V_{\text {us }} / V_{\text {ud }}$ via the ratio $R_{V}=$ $\Gamma(K \rightarrow \pi l \nu(\gamma)) / \Gamma\left(\pi^{+} \rightarrow \pi^{0} e^{+} \nu(\gamma)\right)$ which provides a measure of $f_{+}^{K}(0)\left|V_{\text {us }}\right| / f_{+}^{\pi}(0)\left|V_{\text {ud }}\right|$ independent of the Fermi constant and short-distance radiative corrections. Its dependence on the ratio of two hadronic vector current form factors provides an interesting computational goal for lattice gauge theory studies. Employing a recent lattice based value $f_{+}^{K}(0) / f_{+}^{\pi}(0)=0.970(2)$, we find $V_{\text {us }} / V_{\text {ud }}=0.22910(91)$ compared to $V_{\text {us }} / V_{\text {ud }}=0.23131(45)$ obtained from $R_{A}=\Gamma(K \rightarrow \mu \nu(\gamma)) / \Gamma(\pi \rightarrow \mu \nu(\gamma))$. Those independent $V_{\text {us }} / V_{\text {ud }}$ determinations exhibit a $2.2 \sigma$ discrepancy. That tension suggests a needed shift in the lattice $f_{+}^{K}(0) / f_{+}^{\pi}(0)$ value towards the consistency range $0.961(4)$, experimental/theory input changes or "new physics" effects. Other features and implications of $R_{V}$ and $R_{A}$ are also discussed.

DOI: 10.1103/PhysRevD.101.091301

\section{INTRODUCTION}

The Standard Model (SM) of elementary particle physics includes a three-generation Cabibbo-Kobayashi-Maskawa (CKM) [1,2] $3 \times 3$ quark mixing matrix, $V_{i j}, i=u, c, t$, $j=d, s, b$, which satisfies unitarity, $V^{\dagger}=V^{-1}$. That condition gives rise to orthonormal relationships among its rows and columns. Of special interest is the first row constraint

$$
\left|V_{\mathrm{ud}}\right|^{2}+\left|V_{\mathrm{us}}\right|^{2}+\left|V_{\mathrm{ub}}\right|^{2}=1
$$

Neglecting the tiny $\left|V_{\mathrm{ub}}\right|^{2} \simeq 2 \times 10^{-5}$ contribution [3], it simplifies to approximately the original Cabibbo [1] twogeneration relationship

$$
\left|V_{\text {ud }}\right|^{2}+\left|V_{\text {us }}\right|^{2}=1
$$

An unambiguous experimental deviation from Eq. (2) would signal the presence of "new physics" beyond SM

Published by the American Physical Society under the terms of the Creative Commons Attribution 4.0 International license. Further distribution of this work must maintain attribution to the author(s) and the published article's title, journal citation, and DOI. Funded by SCOAP ${ }^{3}$.
(BSM) expectations. BSM examples include right-handed weak currents, charged Higgs scalars, leptoquarks, $Z^{\prime}$ box diagram loop effects, and exotic muon decay amplitudes that effectively modify the value of the Fermi constant, etc. Such a discovery would have major repercussions; but, first, its acceptance would require evidence of high significance (5 or more sigma effects) as well as confirmation by other measurements.

Up until recently, Eq. (2) appeared to be well satisfied $[3,4]$. However, a novel dispersion relation (DR) approach [5] to super-allowed nuclear beta decay loop effects found an increase in the electroweak radiative corrections that reduced $V_{\text {ud }}$ from $0.97420(21)$ to

$$
\left|V_{\text {ud }}\right|=0.97370(10)_{\mathrm{NP}}(10)_{\mathrm{RC}} \quad \text { DR Result [5], }
$$

where NP (nuclear physics) and RC (radiative corrections) label the uncertainty sources. A subsequent calculation [6] based on a somewhat different approach found

$$
\left|V_{\mathrm{ud}}\right|=0.97389(10)_{\mathrm{NP}}(14)_{\mathrm{RC}} \quad \mathrm{CMS}[6] .
$$

When used with the $V_{\text {us }}$ average [3] from $K_{l 3}(K \rightarrow \pi l \nu)$ and $K_{l 2}\left(K^{ \pm} \rightarrow l^{ \pm} \nu\right)$ decays

$$
\left|V_{\text {us }}\right|=0.2245(8) \quad \text { Error scale factor 2, }
$$


the two approaches lead to roughly 3 and $2 \sigma$ deviations from unitarity respectively. Those discrepancies could be early hints of new physics starting to show up as an effective deviation from CKM unitarity. Alternatively, missing nuclear physics effects and theoretical uncertainties might eventually resolve the problem [7] more conventionally.

The current $V_{\text {us }}$ situation requires some explanation. $K_{l 3}$ decays considered alone give relatively small

$$
\left|V_{\text {us }}\right|=0.2234(8) \quad K_{l 3} \text { decays. }
$$

In contrast, the ratio [8]

$$
R_{A}=\frac{\Gamma(K \rightarrow \mu \nu(\gamma))}{\Gamma(\pi \rightarrow \mu \nu(\gamma))}
$$

is generally considered a more dependable constraint, since common uncertainties in kaon and pion decays tend to cancel in the ratio. That is particularly important for lattice gauge theory input calculations of $f_{K^{+}} / f_{\pi^{+}}$. From the experimental constraint $R_{A}=1.3367(28)$, one finds

$$
\frac{\left|V_{\mathrm{us}}\right| f_{K^{+}}}{\left|V_{\mathrm{ud}}\right| f_{\pi^{+}}}=0.2760(4)
$$

Then using the lattice value [9]

$$
\frac{f_{K^{+}}}{f_{\pi^{+}}}=1.1932(19)
$$

one obtains

$$
\frac{\left|V_{\text {us }}\right|}{\left|V_{\text {ud }}\right|}=0.23131(45)
$$

Agreement with three-generation unitarity requires

$$
\left|V_{\text {ud }}\right|=0.97428(10) \quad \text { and } \quad\left|V_{\text {us }}\right|=0.2253(4) \text {. }
$$

Those SM expectations are 2 or more $\sigma$ different from some of the current $\left|V_{\text {ud }}\right|$ and $\left|V_{\text {us }}\right|$ values shown above. $R_{A}$, being rather free of theoretical and experimental uncertainties, currently represents our best first row CKM constraint and should be taken seriously.

Having set the stage for CKM inconsistencies we now introduce and examine a weak vector current analog of $R_{A}$ for which short-distance electroweak and some part of the quantum electrodynamics (QED) radiative corrections as well as muon lifetime normalization dependence via the Fermi constant cancel in the ratio. Our new vector current analog of $R_{A}$ may also provide a useful target for testing lattice gauge theory calculations. The specific ratio we first consider is

$$
R_{V}=\frac{\Gamma\left(K_{L} \rightarrow \pi^{ \pm} e^{\mp} \nu(\gamma)\right)}{\Gamma\left(\pi^{+} \rightarrow \pi^{0} e^{+} \nu(\gamma)\right)}
$$

which compares radiative inclusive $K_{l 3}$ for the $K_{L}$ and $\pi_{e 3}$ decay rates. The $K_{L}$ is chosen because it is, currently, the best measured. The denominator decay rate is generally viewed as a theoretically pristine method for measuring $V_{\text {ud }}$; but, unfortunately, it is not quantitatively competitive with more precise determinations of that matrix element. Nevertheless, we will show that $R_{V}$ is competitive as a normalization for $K_{e 3}$ and its use for the determination of $V_{\text {us }}$ in much the same way but currently with about a factor of 2 less precision than $R_{A}$. The two ratios are complementary in that one tests the weak charged vector current while the other one probes the axial-vector analog.

\section{PION BETA DECAY}

The branching ratio [10] for pion beta decay, $\pi^{+} \rightarrow$ $\pi^{0} e^{+} \nu(\gamma)$, is $1.038(6) \times 10^{-8}$ where we have taken the liberty to increase the published central value by $+0.2 \%$, in keeping with an updated [3] normalizing $\operatorname{BR}\left(\pi^{+} \rightarrow e^{+} \nu(\gamma)\right)=1.2325(23) \times 10^{-4}$. Used in conjunction with the pion lifetime $26.033(5) \times 10^{-9} \mathrm{~s}$, it implies the experimental decay rate

$$
\Gamma\left(\pi^{+} \rightarrow \pi^{0} e^{+} \nu(\gamma)\right)=0.3988(23) \mathrm{s}^{-1},
$$

which can be compared with the rather precise SM theoretical prediction [11-14]

$\Gamma\left(\pi^{+} \rightarrow \pi^{0} e^{+} \nu(\gamma)\right)=\frac{G_{\mu}^{2}\left|V_{\mathrm{ud}}\right|^{2} m_{\pi^{+}}^{5}\left|f_{+}^{\pi}(0)\right|^{2}}{64 \pi^{3}}\left(1+\mathrm{RC}_{\pi}\right) I_{\pi}$,

where $[11,12]$

$$
\begin{aligned}
\mathrm{RC}_{\pi}= & 0.0334(10), \\
I_{\pi}= & \frac{32}{15}\left(1-\frac{\Delta}{2 m_{\pi^{+}}}\right)^{3}\left(\frac{\Delta}{m_{\pi^{+}}}\right)^{5} f(\epsilon, \Delta) \\
= & 7.376(1) \times 10^{-8}, \\
f(\epsilon, \Delta)= & \sqrt{1-\epsilon}\left(1-\frac{9 \epsilon}{2}-4 \epsilon^{2}\right)+\frac{15}{2} \epsilon^{2} \ln \frac{1+\sqrt{1-\epsilon}}{\sqrt{\epsilon}} \\
& -\frac{3}{7} \frac{\Delta^{2}}{\left(m_{\pi^{+}}+m_{\pi^{0}}\right)^{2}}, \\
\Delta= & m_{\pi^{+}}-m_{\pi^{0}}, \quad \epsilon=m_{e}^{2} / \Delta^{2},
\end{aligned}
$$

where the $+0.0334(10)$ represents our estimate of the electroweak and quantum electrodynamics radiative corrections and their uncertainty [13-17]. Its relatively small theoretical uncertainty is justified by the good agreement 
between the current algebra $[12,14]$ and chiral perturbation theory [13] calculations of the radiative corrections. Both include a universal short-distance 0.0234 electroweak correction combined with a 0.010(1) long-distance QED contribution. Solving for $V_{\text {ud }}$ leads to

$$
\left|V_{\text {ud }}\right|=0.9739(29) \text {. }
$$

That value is in good accord with expectations from CKM unitarity, but it is not competitive with super-allowed nuclear beta decays which are more precise by better than an order of magnitude [4]. Even further improvement by an additional factor of 2 or 3, which appears to be possible but challenging [15], would not make pion beta decay directly competitive for determining $V_{\text {ud }}$. However, we note that currently, the $\pm 0.6 \%$ fractional uncertainty in the pion beta decay rate [see Eq. (13)] is similar to individual $K_{l 3}$ rates used in the determinations of $V_{\text {us }}$. So, it can be used to normalize $K_{l 3}$ decay widths without a significant increase in the overall uncertainty and allows the potential for further improvement. Those features provide the basis for our following discussion.

\section{THE RATIO $R_{V}$}

We begin by considering the $K_{L}(3 e)$ partial decay width, traditionally normalized in terms of the muon lifetime derived Fermi constant, $G_{\mu}$. It has a form similar to Eq. (14),

$\Gamma\left(K_{L} \rightarrow \pi^{\mp} e^{ \pm} \nu(\gamma)\right)=\frac{G_{\mu}^{2}\left|V_{\mathrm{us}}\right|^{2} m_{K_{L}}^{5}\left|f_{+}^{K}(0)\right|^{2}}{192 \pi^{3}}\left(1+\mathrm{RC}_{K}\right) I_{K}$,

with [18]

$$
\begin{aligned}
\mathrm{RC}_{K}= & 0.0334(20), \\
I_{K}= & 2\left(\frac{m_{\pi^{+}}}{m_{K_{L}}}\right)^{4}\left(1+2 \lambda_{+} \frac{m_{K_{L}}^{2}+m_{\pi^{+}}^{2}}{m_{\pi^{+}}^{2}}\right) \\
& \times\left[\frac{\beta_{m}\left(5 \beta_{m}^{2}-3\right) E_{m}^{4}}{m_{\pi^{+}}^{4}}+3 \ln \frac{E_{m}\left(1+\beta_{m}\right)}{m_{\pi^{+}}}\right]-\frac{64 \lambda_{+} \beta_{m}^{5} E_{m}^{5}}{5 m_{\pi^{+}}^{2} m_{K_{L}}^{3}} \\
= & 0.15455(15) \text { and } \beta_{m}=\sqrt{1-\left(m_{\pi^{+}} / E_{m}\right)^{2}}
\end{aligned}
$$

where $E_{m}=0.26838 \mathrm{GeV}$ is the maximum pion energy in the $K_{L}$ rest frame and $\lambda_{+}=0.0282(4)$ parametrizes the average linear energy dependence (slope) of the form factor $f_{+}^{K}$ [3]. Although we employ only the average $\lambda_{+}$term in the form factor expansion for illustrative simplicity, the $I_{K}$ value found is very close, within (roughly) $1 \sigma$, to the result for $I_{K}$ obtained by keeping higher order terms in the form factor expansion that is fit to the physical decay spectrum. Alternatively, $I_{K}$ can be obtained using a dispersive approach. We actually employ the updated dispersive value $I_{K}=0.15470(15)$ [19] in our following analysis. The radiative corrections $\left(\mathrm{RC}_{\pi, K}\right)$ in Eqs. (14) and (20) are to a very good approximation equal in magnitude and cancel (up to the uncertainties) in the ratio, $R_{V}$, defined in Eq. (12),

$$
\frac{1+\mathrm{RC}_{K}}{1+\mathrm{RC}_{\pi}} \simeq 1+\mathrm{RC}_{K}-\mathrm{RC}_{\pi}=1.000(2)_{K}(1)_{\pi} .
$$

Cancellation includes common uncertainties in the shortdistance electroweak and QED radiative corrections. Shortdistance cancellation is expected, but the QED longdistance 0.010(2) cancellation with $0.010(1)$ appears to be accidental. Long-distance SM effects for other $K$ decay modes will differ somewhat, numerically $[18,20]$ from $\mathrm{RC}_{K}=0.0334(20)$ and do not fully cancel. Their remainders and correlated theory uncertainties are included as part of the $K$ contribution when we average over all $K_{l 3}$ decay modes. The current underlying radiative correction uncertainty is estimated to be about $0.2 \%$ at the decay rate level [20]. It represents a theoretical limitation for future $K_{l 3}$ extractions of $V_{\text {us }}$. We note, however, that further reduction in the radiative corrections uncertainty may be possible using the current algebra formalism [12] for $K_{l 3}$ decays as a check on chiral perturbation theory, an approach recently advocated by Seng, Galviz and Meißner [21]. It could, in principle, allow one to refine the calculation of $\mathrm{RC}_{\pi}-\mathrm{RC}_{K}$ and compare with the values we have taken from $[12,14,18,20]$. Given the current excellent agreement among the different $K_{l 3}$ decay modes, we do not expect major changes, but better precision along with a check of the long-distance QED corrections would be welcome. At present, experimental improvement in $R_{V}$ up to about a factor of 3 can be envisioned before confronting theoretical uncertainty in the radiative corrections.

The usual method for extracting $V_{\text {us }}$ is to compare the $K_{L}(3 e)$ partial width theory with experiment to obtain the constraint $f_{+}^{K}(0) V_{\text {us }}=0.2165(6)$. Employing a lattice gauge theory calculation for the form factor is then used to determine $V_{\text {us }}$ at about the $0.3 \%$ level. Its value depends on the form factor as well as factorized electroweak shortdistance and QED long-distance radiative corrections along with the Fermi constant, all of theoretical origin and assumed to be consistent with the definitions of $V_{\text {us }}$ and $f_{+}^{K}(0)$ employed. Next, as an alternative, we normalize relative to pion beta decay which provides a different perspective on testing CKM unitarity and a means to search for the presence of new physics. Of course, a similar exercise can be carried out for any of the $K_{l 3}$ neutral and charged kaon decay modes. Then, the results (including correlated errors) can be averaged to somewhat reduce the uncertainties.

The ratio $R_{V}$, defined in Eq. (12), has the current experimental value 


$$
\begin{aligned}
R_{V}^{\mathrm{exp}} & =\frac{\tau_{\pi} \times \mathrm{BR}\left(K_{L} \rightarrow \pi^{ \pm} e^{\mp} \nu(\gamma)\right)}{\tau_{K_{L}} \times \mathrm{BR}\left(\pi^{+} \rightarrow \pi^{0} e^{+} \nu(\gamma)\right)} \\
& =\frac{26.033(5) \mathrm{ns} \times 0.4056(9)}{51.16(21) \mathrm{ns} \times 1.038(6) \times 10^{-8}} \\
& =1.9884(115)(93) \times 10^{7},
\end{aligned}
$$

where the first uncertainty stems from the pion partial width and the second from the $K_{L}$ lifetime and branching ratio. The latter can be reduced by roughly a factor of $2 / 3$ by averaging over all $K_{l 3}$ partial widths after accounting for differences in phase space, QED corrections, particle masses, a second form factor for muon modes and, in the case of charged kaons, strong isospin breaking [20,22,23].

The experimental value of Eq. (24) should be compared with the SM prediction $[18,19,22-25]$

$R_{V}^{\text {theory }}=\frac{1}{3}\left(\frac{m_{K^{0}}}{m_{\pi^{+}}}\right)^{5}\left(\frac{f_{+}^{K}(0)}{f_{+}^{\pi}(0)} \frac{V_{\mathrm{us}}}{V_{\mathrm{ud}}}\right)^{2} \times \frac{I_{K}}{I_{\pi}} \times 1.000(2)$.

Equating theory and experiment leads to

$$
\frac{f_{+}^{K}(0)}{f_{+}^{\pi}(0)} \frac{V_{\mathrm{us}}}{V_{\mathrm{ud}}}=0.22220(64)(58),
$$

with the first and second errors coming from the pion and kaon decay modes respectively. The second error (58) in Eq. (26) includes uncertainties from $I_{K}$ and $\mathrm{RC}_{K}$. Together they contribute about (22) to the (58). The radiative corrections (modulo uncertainties) $\left(\mathrm{RC}_{\pi, K}\right)$ and Fermi constant have canceled out in the ratio, $I_{K}$ and $I_{\pi}$ are phase space integrals and $f_{+}^{K}(0)$ is the $K \pi$ vector transition form factor, which is 1 up to second order in SU(3) flavor breaking [26] for the neutral kaon. [The charged kaon form factor requires [27] about a 2.8(3)\% increase to fully account for its difference with the neutral kaon case. That shift is consistent with expectations due to strong isospin breaking resulting from pion-eta mixing.] The pion form factor, $f_{+}^{\pi}(0)$, is essentially 1 in the $\mathrm{SU}(2)$ flavor limit [28]. The deviation from 1 is $\mathcal{O}\left(10^{-5}\right)$ and can usually be ignored. However, we retain the form factor ratio $f_{+}^{K}(0) / f_{+}^{\pi}(0)$ in our discussion, since for some lattice calculations that ratio may provide a means for extraneous lattice artifacts, such as finite size effects, to cancel.

Equating $R_{V}$ experiment and theory followed by weighted averaging overall $K_{l 3}$ modes, allowing for correlated uncertainties, as shown in Table I, and following the literature cited, our analysis roughly corresponds to rescaling the carefully studied 5 known $f_{+}^{K}(0)\left|V_{\text {us }}\right|$ values. It leads to the average

$$
\frac{f_{+}^{K}(0) V_{\mathrm{us}}}{f_{+}^{\pi}(0) V_{\mathrm{ud}}}=0.22223(64)(40),
$$

TABLE I. $\quad K_{l 3}$ results from five decay modes with approximate errors, weighted average (including some correlated theory uncertainties) for $f_{+}^{K}(0)\left|V_{\text {us }}\right| / f_{+}^{\pi}(0)\left|V_{\text {ud }}\right|[18,20]$ based in part on the updated results in $[19,25]$. Also shown are the individual $f_{+}^{K}(0) / f_{+}^{\pi}(0)$ values and their average for $\left|V_{\mathrm{us}}\right| /\left|V_{\mathrm{ud}}\right|=$ $0.23131(45)$ [see Eq. (10)].

\begin{tabular}{llc}
\hline \hline Decay & \multicolumn{1}{c}{$\frac{f_{+}^{K}(0)\left|V_{\mathrm{us}}\right|}{f_{+}^{\pi}(0)\left|V_{\mathrm{ud}}\right|}$} & \multicolumn{1}{c}{$\frac{f_{+}^{K}(0)}{f_{+}^{\pi}(0)}$} \\
\hline$K_{L}(e 3)$ & $0.22220(64)(58)$ & $0.9606(28)(19)(25)$ \\
$K_{L}(\mu 3)$ & $0.22250(64)(64)$ & $0.9619(28)(19)(28)$ \\
$K_{S}(e 3)$ & $0.22138(64)(134)$ & $0.9571(28)(19)(52)$ \\
$K^{ \pm}(e 3)$ & $0.22220(64)(86)$ & $0.9606(28)(19)(37)$ \\
$K^{ \pm}(\mu 3)$ & $0.22200(64)(111)$ & $0.9602(28)(19)(48)$ \\
Average & $0.22223(64)(40)$ & $0.9607(28)(19)(18)$ \\
\hline \hline
\end{tabular}

where the central value has remained nearly unchanged, but the kaon dependent uncertainty has been reduced by about a factor of $2 / 3$. We note that the $\chi^{2} /$ degree of freedom for the five $K_{l 3}$ modes was found $[19,25]$ to be an acceptable $0.98 / 4$; so it is not necessary to scale up the error. The goodness of the fit also helps validate the relative magnitude of the radiative corrections applied to the different $K$ decay modes.

Requiring $R_{V}$ and $R_{A}$ to have the same $V_{\text {us }} / V_{\text {ud }}=$ $0.23131(45)$ within errors implies $f_{+}^{K}(0) / f_{+}^{\pi}(0)=$ $0.9607(38)$. For $f_{+}^{\pi}(0)=1$ the implied $f_{+}^{K}(0)=0.9607(38)$ is in tension with the prevailing [29] lattice result of roughly $0.970(2)$ for $2+1+1$ quark flavors by about $2.2 \sigma$. That discrepancy can also be illustrated by inserting $f_{+}^{K}(0) / f_{+}^{\pi}(0)=0.970(2)$ in Eq. (27) which leads to $V_{\text {us }} / V_{\text {ud }}=0.22910(91)$. It differs from Eq. (10) by a related $2.2 \sigma$. If we only used $K_{L}(e 3)$ in our comparison, the $2.2 \sigma$ difference would have been $2.0 \sigma$. Averaging over the five decay modes has not had a dramatic effect. It does, however, demonstrate the consistency of our experimental and theoretical input.

The roughly $2 \sigma$ discrepancy was already observed some time ago in global $K$ decay fits [18]. Currently, averaging over the five kaon beta decay modes indicates about a $2.6 \sigma$ discrepancy with the unitarity based $R_{A}=0.23131(45)$ constraint for $V_{\text {us }}$. An alternative interpretation of the apparent lattice value discrepancy is the need for an additional roughly -0.01 electromagnetic radiative correction relating electromagnetically free $f_{+}^{K}(0)=0.97$ obtained from pure lattice quantum chromodynamics with the form factor appropriate for the decay rate formula in Eq. (20). Such an effect would increase the central value of $V_{\text {us }}$ to 0.2253 , consistent with $R_{A}$ and CKM unitarity. If instead new physics is responsible for the discrepancy, it is less likely due to short-distance electroweak radiative corrections, muon lifetime $G_{\mu}$ normalization or some other effect common to the numerator and denominator which cancels in the $R_{V}$ ratio. We note that a similar discrepancy occurs in $2+1$ flavor lattice gauge theories which predict 
$f_{+}^{K}(0)=0.968$, supporting the interpretation that a reduction in the lattice form factor by about -0.01 may be the most likely route to CKM unitarity. To resolve this situation, additional lattice studies and scrutiny are needed.

We also note that employing $V_{\text {us }} / V_{\text {ud }}=0.22910(102)$ suggested from $R_{V}$, lattice $f_{+}^{K}(0)=0.970(2)$ plus three-generation unitarity implies $V_{\text {ud }}=0.97474(22)$ which exceeds Eq. (11). That makes it more difficult to reconcile with the current super-allowed nuclear beta decay discrepancy.

We averaged over all five $K_{l 3}$ decay modes to reduce the second error in Eq. (26) by roughly a factor of 2/3. Future improvements in kaon measurements are expected [18,23] to further reduce that part of the uncertainty by another $2 / 3$, leaving the partial width of pion beta decay as the dominant uncertainty in the error budget by about a factor of 2.5 . Improving the experimental pion partial beta decay width by a factor of 2 to 3 would bring the overall experimental error budget for $R_{V}$ down by roughly a factor of 2 . At that level, averaging over kaon modes becomes more important. Such a reduction in the $V_{\text {us }} / V_{\text {ud }}$ uncertainty derived from $R_{V}$ along with a similar improvement in $R_{A}$ will together strongly restrict or provide evidence for the existence of new physics at potentially high significance. The latter scenario would be more likely if the $V_{\text {ud }}$ from superallowed beta decays continues to show a deviation from CKM unitarity. Neutron lifetime and decay asymmetry precision measurements should also help resolve the $V_{\text {ud }}$ problem [6,30]. Indeed, the larger radiative corrections found in $[5,6]$ combined with a unitarity favored 0.97428 for $\left|V_{\mathrm{ud}}\right|$ and 1.2762 for $g_{A}$ predict a neutron lifetime of about $878 \mathrm{~s}$ with a small uncertainty.

More precise experimental measurements are clearly needed to reconcile CKM unitarity or unveil evidence for new physics. Our study of pion beta decay and the utility of $R_{V}$ will hopefully reinvigorate interest in that experimental effort. More specifically, on the basis of its complementary role, we advocate a new experiment on pion beta decay designed to improve measurement of that rare branching ratio by an overall factor of 2 to 3 [10]. At that level, combined with anticipated $K_{l 3}$ improvements, it could provide the best determination of $V_{\text {us }}$ or at least a consistency check on lattice calculations. In addition, we encourage the lattice gauge theory community to examine the possibility of a reduced uncertainty to 0.001 and check for a difference in the definition of the lattice $f_{+}^{K}(0)$ and the form factor as defined by $R_{A}$.

\section{ACKNOWLEDGMENTS}

The work of A. C. was supported by the Natural Sciences and Engineering Research Council of Canada. The work of W. J. M. was supported by the U.S. Department of Energy under Grant No. DE-SC0012704. The work of A. S. was supported in part by the National Science Foundation under Grant No. PHY-1620039. This manuscript has been coauthored by employees of Brookhaven Science Associates, LLC under Contract No. DE-SC0012704 with the U.S. Department of Energy. The publisher by accepting the manuscript for publication acknowledges that the U.S. Government retains a non-exclusive, paid-up, irrevocable, world-wide license to publish or reproduce the published form of this manuscript, or allow others to do so, for U.S. Government purposes. This preprint is intended for publication in a journal or proceedings. Since changes may be made before publication, it may not be cited or reproduced without the author's permission. This report was prepared as an account of work sponsored by an agency of the U.S. Government. Neither the U.S. Government nor any agency thereof, nor any of their employees, nor any of their contractors, subcontractors, or their employees, makes any warranty, express or implied, or assumes any legal liability or responsibility for the accuracy, completeness, or any third party's use or the results of such use of any information, apparatus, product, or process disclosed, or represents that its use would not infringe privately owned rights. The views and opinions of authors expressed herein do not necessarily state or reflect those of the U.S. Government or any agency thereof.

Note Added.-After our paper was submitted for publication, a preprint by the PACS Collaboration appeared [31]. It finds for a large lattice with $2+1$ quark flavors, $f_{+}^{K}(0)=0.960(5)$. Also, a paper by X. Feng et al. [32] was posted on the arXiv. It confirms our calculation of the pion beta decay rate using a novel lattice approach and reduces the theoretical uncertainty by a factor of 3 , strengthening our result and its call for an improved experimental measurement of that rare decay mode. 
[1] N. Cabibbo, Unitary Symmetry and Leptonic Decays, Phys. Rev. Lett. 10, 531 (1963).

[2] M. Kobayashi and T. Maskawa, $C P$ violation in the renormalizable theory of weak interaction, Prog. Theor. Phys. 49, 652 (1973).

[3] M. Tanabashi et al., Review of particle physics, Phys. Rev. D 98, 030001 (2018).

[4] J. Hardy and I. S. Towner, $\left|V_{u d}\right|$ from nuclear $\beta$ decays, Proc. Sci., CKM2016 (2016) 028.

[5] C.-Y. Seng, M. Gorchtein, H. H. Patel, and M. J. RamseyMusolf, Reduced Hadronic Uncertainty in the Determination of $V_{u d}$, Phys. Rev. Lett. 121, 241804 (2018).

[6] A. Czarnecki, W. J. Marciano, and A. Sirlin, Radiative corrections to neutron and nuclear beta decays revisited, Phys. Rev. D 100, 073008 (2019).

[7] C. Y. Seng, M. Gorchtein, and M. J. Ramsey-Musolf, Dispersive evaluation of the inner radiative correction in neutron and nuclear $\beta$ decay, Phys. Rev. D 100, 013001 (2019).

[8] W. J. Marciano, Precise Determination of $\left|V_{\text {us }}\right|$ from Lattice Calculations of Pseudoscalar Decay Constants, Phys. Rev. Lett. 93, 231803 (2004).

[9] S. Aoki et al., FLAG review 2019, Eur. Phys. J. C 80, 113 (2020).

[10] D. Pocanic et al., Precise Measurement of the $\pi^{+} \rightarrow \pi^{0} e^{+} \nu$ Branching Ratio, Phys. Rev. Lett. 93, 181803 (2004).

[11] G. Källén, Elementary Particle Physics (Addison-Wesley, Reading, MA, 1964).

[12] A. Sirlin, Current algebra formulation of radiative corrections in gauge theories and the universality of the weak interactions, Rev. Mod. Phys. 50, 573 (1978); Erratum, Rev. Mod. Phys. 50, 905(E) (1978).

[13] V. Cirigliano, M. Knecht, H. Neufeld, and H. Pichl, The Pionic beta decay in chiral perturbation theory, Eur. Phys. J. C 27, 255 (2003).

[14] M. Passera, K. Philippides, and A. Sirlin, Observations on the radiative corrections to pion $\beta$-decay, Phys. Rev. D 84, 094030 (2011).

[15] W. McFarlane, PILAC Users Group Report No. 1A-112-92180, 1994.

[16] V. Cirigliano, $K(e 3)$ and $\pi(e 3)$ decays: Radiative corrections and CKM unitarity, arXiv:hep-ph/0305154.

[17] M. Knecht, Chiral perturbation theory confronted with experiment, Frascati Phys. Ser. 36, 397 (2004).

[18] M. Antonelli et al., An Evaluation of $\left|V_{u s}\right|$ and precise tests of the Standard Model from world data on leptonic and semileptonic kaon decays, Eur. Phys. J. C 69, 399 (2010).

[19] V. Cirigliano, E. Passemar, and M. Moulson, Extraction of $V_{u s}$ from experimental measurements, Proceedings of the International Conference on Kaon Physics 2019 (2019), https://indico.cern.ch/event/769729/contributions/3512047/ attachments/1905114/3146148/Kaon2019_MoulsonPassemar Corr.pdf.

[20] V. Cirigliano, M. Giannotti, and H. Neufeld, Electromagnetic effects in $K_{l 3}$ decays, J. High Energy Phys. 11 (2008) 006.

[21] C.-Y. Seng, D. Galviz, and U.-G. Meißner, A new theory framework for the electroweak radiative corrections in $K_{l 3}$ Decays, J. High Energy Phys. 02 (2020) 069.

[22] V. Cirigliano, M. Knecht, H. Neufeld, H. Rupertsberger, and P. Talavera, Radiative corrections to $K_{l 3}$ decays, Eur. Phys. J. C 23, 121 (2002).

[23] V. Cirigliano, Precision tests of the standard model with $K_{l 3}$ decays, Proc. Sci., KAON (2008) 007.

[24] M. Moulson, Experimental determination of $V_{u s}$ from kaon decays, Proc. Sci., CKM2016 (2017) 033 [arXiv:1704.04104].

[25] V. Cirigliano, M. Moulson, and E. Passemar, The status of $V_{u s}$ (Current and future status of first-row CKM unitarity), Proceedings of the Amherst MA on 2019 (2019), https://www .physics.umass.edu/acfi/seminars-and-workshops/currentand-future-status-of-the-first-row-ckm-unitarity/1147.

[26] M. Ademollo and R. Gatto, Nonrenormalization Theorem for the Strangeness Violating Vector Currents, Phys. Rev. Lett. 13, 264 (1964).

[27] H. Leutwyler and M. Roos, Determination of the elements $\mathrm{V}(\mathrm{us})$ and $\mathrm{V}(\mathrm{ud})$ of the Kobayashi-Maskawa matrix, Z. Phys. C 25, 91 (1984).

[28] R. E. Behrends and A. Sirlin, Effect of Mass Splittings on the Conserved Vector Current, Phys. Rev. Lett. 4, 186 (1960).

[29] A. Bazavov et al., $\left|V_{u s}\right|$ from $K_{\ell 3}$ decay and four-flavor lattice QCD, Phys. Rev. D 99, 114509 (2019).

[30] A. Czarnecki, W. J. Marciano, and A. Sirlin, Neutron Lifetime and Axial Coupling Connection, Phys. Rev. Lett. 120, 202002 (2018).

[31] J. Kakazu, K.-I. Ishikawa, N. Ishizuka, Y. Kuramashi, Y. Nakamura, Y. Namekawa, Y. Taniguchi, N. Ukita, T. Yamazaki, and T. Yoshié, $K_{l 3}$ form factors at the physical point on $(10.9 \mathrm{fm})^{3}$ volume, arXiv:1912.13127.

[32] X. Feng, M. Gorchtein, P.-X. Ma, L.-C. Jin, and C.-Y. Seng, First-principles calculation of electroweak box diagrams from lattice QCD, Phys. Rev. Lett. 124, 192002 (2020). 